\title{
Integration of Pre-intubation Ultrasound into Airway Management Course: A Novel Training Program
}

\author{
Srikar Adhikari ${ }^{1}$, Elaine Situ-LaCasse ${ }^{2}$, Josie Acuña ${ }^{3}$, Steven Irving ${ }^{4}$, Christina Weaver ${ }^{5}$, Kara Samsel ${ }^{6}$, David E Biffar ${ }^{7}$,
} Mahsaw Motlagh ${ }^{8}$, John Sakles ${ }^{9}$

\begin{abstract}
Objectives: To determine the feasibility of integrating pre-intubation ultrasound into airway course and assess emergency medicine (EM) residents' confidence and comfort level in using ultrasound for pre-intubation hemodynamic stabilization and identifying cricothyroid membrane after the training session.

Materials and methods: This is a retrospective study. Pre-intubation ultrasound training was delivered with the following ultrasound components (didactics and hands-on sessions using human models) to EM residents: (1) sonoanatomy and scanning technique to identify cricothyroid membrane and (2) pre-intubation echocardiography for recognition of acute right ventricular failure and pre-intubation hemodynamic stabilization.

Results: A total of 56 EM residents participated in this study. Only 21\% [95\% confidence interval (CI), 10-31\%] reported using ultrasound for pre-intubation hemodynamic stabilization. After the training session, $89 \%$ (95\% Cl, 81-97\%) reported that ultrasound-based teaching increased their knowledge of pre-intubation hemodynamic stabilization compared with traditional teaching methods. On a scale of 1 (low) through 10 (high), the average comfort level for integrating ultrasound findings into medical decision making for pre-intubation hemodynamic stabilization was $6.8(95 \% \mathrm{Cl}, 6.3-7.3)$. Seventy-nine percent $(95 \% \mathrm{Cl}, 68-89 \%)$ reported that focused training in airway ultrasound is adequate to identify cricothyroid membrane. On a scale of 1 (low) through 10 (high), the average confidence level for identifying cricothyroid membrane using ultrasound was $6.6(95 \% \mathrm{Cl}, 6.1-7.1)$.

Conclusion: At our institution, we successfully integrated pre-intubation ultrasound into an airway course. Emergency medicine residents had a moderate level of comfort and confidence level using ultrasound for pre-intubation hemodynamic stabilization and identifying cricothyroid membrane after the training session.
\end{abstract}

Keywords: Airway, Cricothyroid membrane, Echocardiography, Emergency medicine, Hemodynamic, Pre-intubation, Ultrasound.

Indian Journal of Critical Care Medicine (2020): 10.5005/jp-journals-10071-23370

\section{INTRODUCTION}

Sonography has recently evolved as a simple, portable, and noninvasive tool for assessment and management of the airway. ${ }^{1}$ Ultrasound allows accurate identification of upper airway anatomical structures such as thyroid cartilage, epiglottis, cricoid cartilage, cricothyroid membrane, tracheal cartilages, and esophagus. ${ }^{2,3}$ In recent years, numerous studies have demonstrated various roles of ultrasound in airway management in a variety of clinical settings. ${ }^{4,5}$ Ultrasound has been shown to be not only useful in diagnosing airway pathology but can also assist in a variety of airway procedures such as cricothyrotomy, tracheostomy, and endotracheal intubation. ${ }^{6-8}$ It facilitates assessment of airway to predict difficult laryngoscopy. ${ }^{9}$ Ultrasound has been found to be a reliable tool for confirmation of endotracheal tube placement and predicting extubation failure. .,10 $^{2}$

Traditionally, cricothyrotomy is performed by palpating anatomic landmarks in the neck, and the success of this procedure is largely dependent on accurately identifying relevant anatomical landmarks (cricothyroid membrane, cricoid, and thyroid cartilages) on palpation. However, palpation of surface landmarks is not always feasible, which can result in multiple unsuccessful attempts and hypoxia in emergent situations. The superiority of ultrasound over digital palpation in identifying cricothyroid membrane has been well documented in the literature. ${ }^{11,12}$ Since cricothyrotomy is a time-sensitive, lifesaving procedure, and successful outcome is dependent on rapid and accurate identification of the cricothyroid
1-4,9 Department of Emergency Medicine, The University of Arizona, Tucson, Arizona, USA

${ }^{5}$ AT Still University School of Osteopathic Medicine, Mesa, Arizona, USA ${ }^{6}$ Department of Emergency Medicine, Texas Tech University Health Sciences Center, El Paso, Texas, USA

${ }^{7}$ Arizona Simulation Technology and Education Center, University of Arizona College of Medicine, Tucson, Arizona, USA

${ }^{8}$ University of Arizona College of Medicine, Tucson, Arizona, USA

Corresponding Author: Srikar Adhikari, Department of Emergency Medicine, The University of Arizona, Tucson, Arizona, USA, Phone: +1520-621-3623, e-mail: sriadhikari@aol.com

How to cite this article: Adhikari S, Situ-LaCasse E, Acuña J, Irving S, Weaver C, Samsel K, et al. Integration of Pre-intubation Ultrasound into Airway Management Course: A Novel Training Program. Indian J Crit Care Med 2020;24(3):179-183.

Source of support: Nil

Conflict of interest: None

membrane, pre-intubation scanning is recommended to identify cricothyroid membrane on ultrasound. ${ }^{13}$ Marking the cricothyroid membrane before an anticipated difficult intubation increases safety, operator confidence, and decreases time to perform the procedure if necessary.

While ultrasound has been found to be useful in identifying and evaluating sonoanatomy of the upper airway, it can also 
play a major role in hemodynamic stabilization of critically ill patients prior to endotracheal intubation. Higgs et al. published guidelines for the management of endotracheal intubation in critically ill patients in 2018. Approximately $6 \%$ of intensive care unit (ICU) patients have a predicted difficult airway. ${ }^{14}$ In addition, critically ill patients are at a high risk of circulatory collapse during endotracheal intubation. There is up to a $25 \%$ risk of hemodynamic instability even in successful ICU intubations. It is recommended to include a cardiovascular component in the protocol during intubation of ICU patients, by defining volume responsiveness and early administration of vasopressors to prevent hemodynamic collapse. ${ }^{14-16}$ Optimizing the hemodynamics prior to intubation is crucial to prevent cardiovascular complications in the periintubation period. The information obtained by performing a brief bedside cardiac ultrasound can change the management of critically ill patient, preventing potential decompensation during and following endotracheal intubation. Intubation of patients with right ventricular heart failure can be catastrophic since the right ventricle is especially sensitive to increases in afterload. In addition, a failing right ventricle may not be able to maintain cardiac output with decreased preload from increased mean airway pressure after intubation. ${ }^{14}$ Pre-intubation cardiac ultrasound will allow clinicians to appropriately prepare for hemodynamic deterioration. Sonographic assessment allows clinicians to approach pre-intubation resuscitation in a specific, targeted manner, such as starting vasopressors, administering afterloadreducing medications, avoiding intubation, etc.

Airway management is a core skill taught and reinforced during residency training, and a variety of refresher courses are offered across the country to train clinicians working both in academic and community settings. However, pre-intubation ultrasound is not commonly taught as a component of airway management courses. To our knowledge, the integration of pre-intubation ultrasound into airway management course is novel and has never been studied. Our objectives were to determine the feasibility of integrating pre-intubation ultrasound into an airway management course and assess emergency medicine (EM) residents' confidence and comfort level in using ultrasound for pre-intubation hemodynamic stabilization and identifying cricothyroid membrane after the training session.

\section{Materials and Methods}

\section{Study Design/Setting/Participants}

This retrospective study was conducted at two academic medical centers with two Accreditation Council for Graduate Medical Education-accredited categorical 3-year EM residency programs and one combined 5-year pediatric/EM residency program. There is a robust ultrasound training program for residents and faculty including an emergency ultrasound fellowship program. The data were collected from EM residents trained at one of the three residency programs. An institutional review board approval was obtained for this study.

\section{Study Protocol}

Airway course is offered to all residents annually at our institution. For each session, there are didactics and hands-on components. A variety of airway techniques are taught during this course, including rigid laryngoscopy (direct laryngoscopy, video laryngoscopy, and bougie), flexible laryngoscopy (flexible fiberoptic scope), rescue oxygenation (facemask ventilation, oropharyngeal airway, nasopharyngeal airway, and supraglottic devices), pediatric airway management, and surgical airway (cricothyrotomy). The course is led by EM faculty members with expertise in airway management.

Integrating pre-intubation ultrasound into the airway course required management of various resources including faculty, human models, and ultrasound equipment. The faculty included emergency physicians with point-of-care ultrasound expertise. Pre-intubation ultrasound was delivered with the following ultrasound components: (1) asynchronous training, (2) didactics, (3) hands-on sessions using human models, and (4) administration of questionnaire.

\section{Asynchronous Learning}

One week before the training session, the course participants received an email with the asynchronous learning materials. The educational elements included ultrasound fundamentals: sonographic anatomy of the upper airway including hyoid bone, thyroid cartilage, cricoid cartilage, cricothyroid membrane, vocal cords, and trachea; upper airway scanning techniques to identify cricothyroid membrane; pre-intubation echocardiography technique including cardiac sonoanatomy; hemodynamic deterioration with intubation in right ventricular failure; recognition of acute right ventricular failure on ultrasound; and pre-intubation hemodynamic stabilization based on ultrasound findings. Participants also received a video link demonstrating cricothyroid membrane identification using ultrasound. The estimated time to review the asynchronous learning materials was approximately 2 hours.

\section{Didactics and Hands-on Session}

On the day of the hands-on session, residents received a brief didactic lecture covering items in the asynchronous learning materials and a review of video clips demonstrating upper airway anatomy and echocardiography findings of right ventricular failure. The ultrasound hands-on training occurred in the simulation center in two stations (upper airway ultrasound and pre-intubation echocardiography) using human models as an integrated component of the airway course. Ultrasound instruction in the upper airway ultrasound station included sonographic anatomy of hyoid bone, thyroid cartilage, cricoid cartilage, cricothyroid membrane, vocal cords, and trachea; scanning technique (transverse and parasagittal planes); and identification of cricothyroid membrane (Fig. 1). In the preintubation echocardiography station, the following items were taught: cardiac sonoanatomy, hemodynamic decompensation with intubation in patients with right ventricular failure, recognition of acute right ventricular failure using ultrasound (sonographic signs of right ventricular failure; Fig. 2), pre-intubation hemodynamic stabilization based on ultrasound findings, and cardiac scanning technique (subxiphoid, parasternal, and apical views). Participants were given the opportunity to practice the techniques on the human models under the supervision of instructors.

\section{Questionnaire}

At the end of the hands-on training session, subjects were asked to complete a questionnaire. The questionnaire consisted of specific questions regarding demographics, prior ultrasound experience, and residents' prior knowledge of pre-intubation hemodynamic stabilization. The questionnaire also included items related to residents' perceptions of focused ultrasound training session to teach upper airway ultrasound to identify cricothyroid membrane 


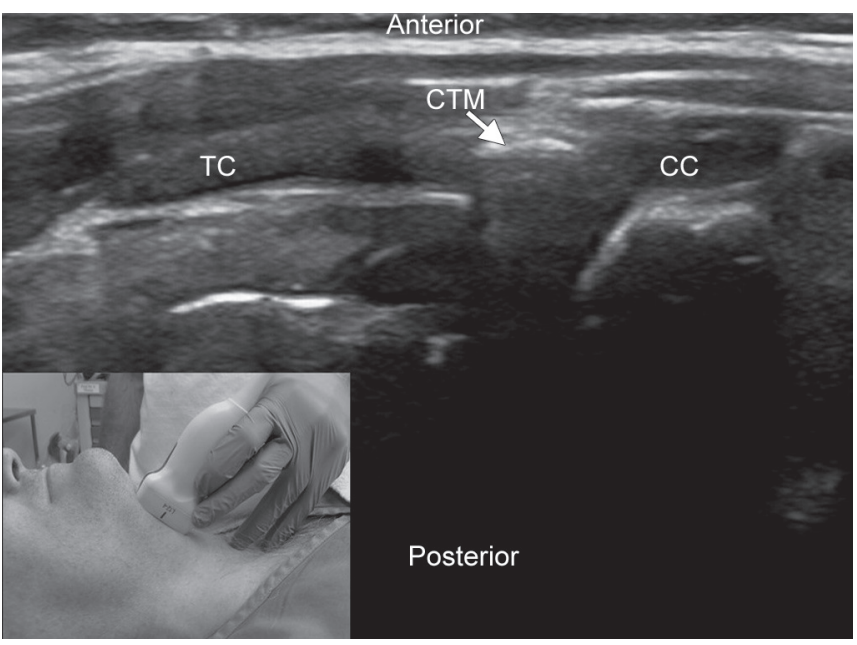

Fig. 1: Cricothyroid membrane seen as a hyperechoic band between the hypoechoic thyroid cartilage and cricoid cartilage in parasagittal plane

and cardiac ultrasound-based pre-intubation hemodynamic stabilization. Questions assessing perceptions were answered on a 5-point Likert-type scale ( 1 = strongly agree, 2 = agree, $3=$ neither agree nor disagree, $4=$ disagree, and $5=$ strongly disagree). The confidence and comfort level of residents in identifying cricothyroid membrane using ultrasound and integrating ultrasound findings into medical decision making for pre-intubation hemodynamic stabilization after the training session were assessed using a 10-point Likert-type response scale ( $1=$ low and $10=$ high). The instructors were responsible for distributing and collecting the questionnaires. The questionnaires were administered and collected anonymously to protect the confidentiality of the subjects. A medical student blinded to the study hypothesis entered all questionnaire data into an Excel database.

\section{Data Analysis}

The data were analyzed using descriptive statistics. Continuous data were presented as averages with standard deviations or $95 \%$ $\mathrm{Cls}$, and dichotomous data and nominal data were presented as a percentage of frequency of occurrences.

\section{Results}

A total of 56 EM residents (35 PGY1s, 12 PGY2s, 7 PGY3s, 1 PGY4, and 1 PGY5) participated in this study. The residents' prior ultrasound experience is summarized in Table 1. Ninety-one percent $(95 \%$ $\mathrm{Cl}, 83-98 \%)$ reported that they are familiar with pre-intubation hemodynamic stabilization and that ultrasound is an important tool to optimize hemodynamics prior to intubation in critically ill patients. However, only $21 \%(95 \% \mathrm{Cl}, 10-31 \%)$ reported using ultrasound for pre-intubation hemodynamic stabilization. Ninety-eight percent agreed that ultrasound is an important tool to optimize hemodynamics prior to intubation in critically ill patients $(95 \% \mathrm{Cl}, 94-101 \%)$. After the training session, $89 \%(95 \% \mathrm{Cl}$, 81-97\%) reported that ultrasound-based teaching increased their knowledge of pre-intubation hemodynamic stabilization compared with traditional teaching methods. On a scale of 1 (low) through 10 (high), the average comfort level for integrating ultrasound findings into medical decision making for pre-intubation hemodynamic stabilization was $6.8(95 \% \mathrm{Cl}, 6.3-7.3)$ after the training session. Seventy-nine percent $(95 \% \mathrm{Cl}, 68-89 \%)$ reported that focused

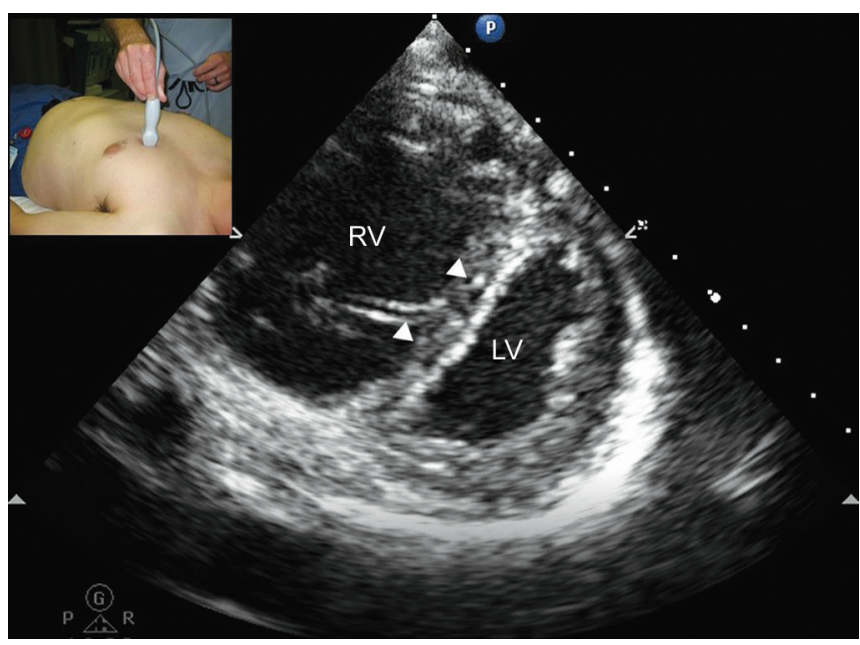

Fig. 2: Parasternal short-axis view demonstrating " $D$ " sign (bowing of the interventricular septum into left ventricle-arrow heads), dilated right ventricle, and small left ventricle

Table 1: Residents' prior ultrasound experience

\begin{tabular}{ll}
\hline Emergency medicine residents $(n)$ & Prior ultrasound examinations $(n)$ \\
\hline 21 & $<50$ \\
5 & $50-100$ \\
11 & $101-150$ \\
7 & $151-200$ \\
12 & $>200$ \\
\hline
\end{tabular}

training in airway ultrasound is adequate to identify cricothyroid membrane. On a scale of 1 (low) through 10 (high), the average confidence level for identifying cricothyroid membrane using ultrasound was $6.6(95 \% \mathrm{Cl}, 6.1-7.1)$. Seventy-five percent $(95 \% \mathrm{Cl}$, $64-86 \%)$ agreed that it is feasible to do rapid airway ultrasound to identify cricothyroid membrane prior to intubation. Ninetyone percent $(95 \% \mathrm{Cl}, 84-98 \%)$ reported that focused ultrasound training (airway ultrasound to identify cricothyroid membrane and ultrasound-based pre-intubation hemodynamic stabilization) should be a part of the airway course.

\section{Discussion}

Our study results suggest that it is feasible to integrate ultrasound into an airway course, and residents can learn these specific ultrasound techniques after a short training session. Majority of the residents (91\%) also reported that focused ultrasound training should be a component of an airway course. We believe that it is critical to teach pre-intubation ultrasound to prevent poor patient outcomes, especially in patients with difficult airway predictors. It is crucial to mark the cricothyroid membrane prior to rapid sequence intubation (RSI) as an integral portion of pre-intubation preparation and protocol. This is particularly useful in patients with risk factors for conversion from RSI to surgical airway: obesity, short neck, facial trauma, etc. This is especially important in the management of critically ill patients, when fluid resuscitation, capillary leak syndromes, prone ventilation, and prolonged intubation can contribute to airway edema and distortion. ${ }^{14}$ External palpation alone for identification of the cricothyroid membrane is challenging, and airway ultrasound allows the physician to rapidly identify and mark the exact location of where the incision is to be made. 
This can prevent several complications, such as trauma to the thyroid, vascular injury, and extension of incisions to find the cricothyroid membrane. Ultrasound has been reported to be superior to palpation in the identification of the cricothyroid membrane even in patients with normal neck anatomy. ${ }^{7}$ Airway management guidelines in the critically ill list identification of the cricothyroid membrane as part of the pre-intubation checklist, and we recommend integrating bedside ultrasound into this process. ${ }^{17}$

The guidelines published by Higgs et al. recommend assigning a team member specifically for monitoring and managing hemodynamic status during the peri-intubation period of a critically ill patient. In addition to the recommended peri-intubation hemodynamic management of intravenous/ intraosseous access and preoxygenation, Higgs et al. warn of periintubation hemodynamic instability, but they make no mention of performing a bedside ultrasound during this critical period before the procedure. ${ }^{14}$ This highly vulnerable population would likely benefit the most from sonographic assessment. Intubation of patients with right ventricle failure may result in the hemodynamic instability. Since a failing right ventricle is very sensitive and unable to compensate for any increase in afterload and decrease in preload from intubation, we recommend to perform pre-intubation echocardiography in critically ill patients to avoid hemodynamic deterioration. Pre-intubation echocardiography will detect signs of right ventricular failure (pressure and volume overload) such as dilated right ventricle, bowing of the interventricular septum into the left ventricle, decrease in the size of left ventricular cavity, and decreased left ventricular filling resulting in decreased cardiac output. Bowing of the interventricular septum into left ventricle is visualized as "D-sign" on a parasternal short-axis view in a patient with right ventricular failure (Fig. 2). ${ }^{18}$ If acute right ventricular failure is detected prior to intubation, it will help choose appropriate management strategy prior to intubation and can avoid hemodynamic deterioration. With pre-intubation detection of right ventricular failure, different ameliorating strategies can be employed prior to the intubation to avoid hemodynamic compromise. Non-invasive positive pressure ventilation could be an alternative in some cases, which has a less pronounced effect on venous return and preload compared with endotracheal intubation. Inhaled nitric oxide can be used to decrease pulmonary artery pressure, especially in the setting of pulmonary arterial hypertension. Other strategies in the setting of right ventricular failure include administration of vasopressors prior to intubation and avoiding fluid boluses.

Even with varied ultrasound experience, our residents had modest confidence after the training session, indicating the feasibility in not only learning these techniques but also integrating them in their clinical practice. However, it is important to identify and address the barriers in routine use. Potential barriers to the use of ultrasound before intubation could be inadequate training (residents and/or faculty), lack of confidence in the technique, accessibility to ultrasound equipment, limitation in space (especially in a crowded room), and lack of integration into the usual preintubation preparation protocol. Integration requires addressing at least the major barriers. Accessibility and space issues could be solved with handheld ultrasounds. Our study only evaluated residents' experience and confidence levels, but protocolization and implementation of this technique requires training of all the physicians, including faculty members. Repeated teaching sessions can bolster confidence and serve as reminders to use ultrasound in their practice. As with any other ultrasound application, performing upper airway ultrasound is operator dependent, requiring sufficient training before confidently incorporating it into practice. With focused training in pre-intubation ultrasound, healthcare providers practicing in acute care settings can reliably use ultrasound in critically ill patients requiring intubation.

After the training session, a majority (89\%) of our subjects reported that ultrasound-based teaching increased their knowledge of pre-intubation hemodynamic stabilization compared with traditional teaching methods. The educational value of incorporating these ultrasound techniques is not limited to the pre-intubation phase. With increased knowledge of cardiac and airway anatomy and physiology, these skills are translated into management and resuscitation of critically ill patients and diagnoses of dangerous airway pathologies. With growing evidence, there is a potential for the incorporation of pre-intubation ultrasound into the future standard of care airway management.

Our study has several limitations including its retrospective nature. Our sample size was small, and the residents were from programs with an established emergency ultrasound fellowship. We had the resources (fellows and faculty, machines, and expertise) to integrate these techniques into the airway management course which may not be available at other institutions. We used a convenience sample of residents which raises the possibility of selection bias. The data were self-reported and are vulnerable to error. All of our questions were closed-ended, which may have also introduced a response bias. We did not assess residents' ultrasound skills after the training session. We did not assess long-term retention skills or the impact of our training on patient outcomes. These hands-on sessions were taught on normal human models which may not reflect the real-world acute care setting environment.

\section{ConClusion}

At our institution, we successfully integrated pre-intubation ultrasound into airway management course. Emergency medicine residents had a moderate level of comfort and confidence level using ultrasound for pre-intubation hemodynamic stabilization and identifying cricothyroid membrane after the training session.

\section{References}

1. Osman A, Sum KM. Role of upper airway ultrasound in airway management. J Intensive Care 2016;4:52. DOI: 10.1186/s40560-0160174-z.

2. Singh M, Chin KJ, Chan VW, Wong DT, Prasad GA, Yu E. Use of sonography for airway assessment: an observational study. J Ultrasound Med 2010;29(1):79-85. DOI: 10.7863/jum.2010.29.1.79.

3. Lun HM, Zhu SY, Liu RC, Gong JG, Liu YL. Investigation of the upper airway anatomy with ultrasound. Ultrasound Q 2016;32(1):86-92. DOI: 10.1097/RUQ.0000000000000163.

4. Kristensen MS, Teoh WH, Graumann O, Laursen CB. Ultrasonography for clinical decision-making and intervention in airway management: from the mouth to the lungs and pleurae. Insights Imaging 2014;5(2):253-279. DOI: 10.1007/s13244-014-0309-5.

5. You-Ten KE, Siddiqui N, Teoh WH, Kristensen MS. Point-ofcare ultrasound (POCUS) of the upper airway. Can J Anaesth 2018;65(4):473-484. DOI: 10.1007/s12630-018-1064-8.

6. Plata P, Gaszyński T. Ultrasound-guided percutaneous tracheostomy. Anaesthesiol Intensive Ther 2019;51(2):126-132. DOI: 10.5114/ ait.2019.86277.

7. Siddiqui N, Arzola C, Friedman Z, Guerina L, You-Ten KE. Ultrasound improves cricothyrotomy success in cadavers with poorly defined 
neck anatomy: a randomized control rrial. Anesthesiology 2015;123(5):1033-1041. DOI: 10.1097/ALN.0000000000000848.

8. Gottlieb M, Holladay D, Peksa GD. Ultrasonography for the confirmation of endotracheal tube intubation: a systematic review and meta-analysis. Ann Emerg Med 2018;72(6):627-636. DOI: 10.1016/j.annemergmed.2018.06.024.

9. Adhikari S, Zeger W, Schmier C, Crum T, Craven A, Frrokaj I, et al. Pilot study to determine the utility of point-of-care ultrasound in the assessment of difficult laryngoscopy. Acad Emerg Med 2011;18(7):754-758. DOI: 10.1111/j.1553-2712.2011.01099.x.

10. Palkar A, Narasimhan M, Greenberg H, Singh K, Koenig S, Mayo $P$, et al. Diaphragm excursion-time index: a new parameter using ultrasonography to predict extubation outcome. Chest 2018;153(5):1213-1220. DOI: 10.1016/j.chest.2018.01.007.

11. Kristensen MS, Teoh WH, Rudolph SS. Ultrasonographic identification of the cricothyroid membrane: best evidence, techniques, and clinical impact. Br J Anaesth 2016;117(Suppl 1):i39-i48. DOI: 10.1093/bja/aew176.

12. Altun D, Ali A, Koltka K, Buget $M$, Çelik M, Doruk C, et al. Role of ultrasonography in determining the cricothyroid membrane localization in the predicted difficult airway. Ulus Travma Acil Cerrahi Derg 2019;25(4):355-360. DOI: 10.14744/tjtes.2019.65250.

13. Mallin M, Curtis K, Dawson M, Ockerse P, Ahern M. Accuracy of ultrasound-guided marking of the cricothyroid membrane before simulated failed intubation. Am J Emerg Med 2014;32(1):61-63. DOI: 10.1016/j.ajem.2013.07.004.
14. Higgs A, McGrath B, Goddard C, Rangasami J, Suntharalingam G, Gale $R$, et al. Guidelines for the management of tracheal intubation in critically ill adults. Br J Anaesth 2018;120(2):323-352. DOI: 10.1016/ j.bja.2017.10.021.

15. Cabrini L, Landoni G, Baiardo Redaelli M, Saleh O, Votta CD, Fominskiy $\mathrm{E}$, et al. Tracheal intubation in critically ill patients: a comprehensive systematic review of randomized trials. Crit Care 2018;22(1):6. DOI: 10.1186/s13054-017-1927-3.

16. Quintard H, I'Her E, Pottecher J, Adnet F, Constantin JM, De Jong A, et al. Experts' guidelines of intubation and extubation of the ICU patient of French Society of Anesthesia and Intensive Care Medicine (SFAR) and French-speaking Intensive Care Society (SRLF): in collaboration with the pediatric Association of French-speaking Anaesthetists and Intensivists (ADARPEF), French-speaking Group of Intensive Care and Paediatric emergencies (GFRUP) and Intensive Care Physiotherapy Society (SKR). Ann Intensive Care 2019;9(1):13. DOI: 10.1186/s13613 019-0483-1.

17. Aslani A, Ng S-C, Hurley M, McCarthy KF, McNicholas M, McCaul CL. Accuracy of identification of the cricothyroid membrane in female subjects using palpation: an observational study. Anesth Analg 2012;114(5):987-992. DOI: 10.1213/ANE.0b013e31824970ba.

18. Tips and Tricks: Intubation in Right Heart Failure, https://www.acep. org/how-we-serve/sections/critical-care-medicine/news/january2017/tips-and-tricks-intubation-in-right-heart-failure/, accessed on July 12,2019 . 